\title{
Control of Acinetobacter baumannii outbreak in the neonatal intensive care unit in Latvia: whole-genome sequencing powered investigation and closure of the ward
}

\author{
A. Gramatniece ${ }^{1,2}$, I. Silamikelis ${ }^{3}$, le. Zahare ${ }^{1}$, V. Urtans ${ }^{1}$, Ir. Zahare ${ }^{1}$, E. Dimina ${ }^{4}$, M. Saule ${ }^{1,2}$, A. Balode ${ }^{1}$, \\ I. Radovica-Spalvina ${ }^{3}$, J. Klovins ${ }^{3}$, D. Fridmanis ${ }^{3}$ and U. Dumpis ${ }^{1,2^{*}}$ (D)
}

\begin{abstract}
Background: Acinetobacter baumannii is an emerging pathogen capable of causing hospital-acquired infections (HAls). It has the ability to survive on environmental surfaces for months, making transmission difficult to control. Our report describes the investigation and restriction of an outbreak of A.baumannii in the Neonatal Intensive Care Unit (NICU) using whole-genome sequencing (WGS) and multi-modal infection control measures.

Methods: A prospective surveillance of HAls was initiated in the NICU at the Pauls Stradins Clinical University Hospital (PSCUH) in Latvia on 1/9/2012 and identified an outbreak of A.baumannii. Case definitions for A.baumannii bloodstream infection (BSI) and colonization were implemented; surveillance cultures were obtained from all admitted patients to monitor the rate of colonization; an infection prevention and control team was formed and infection control interventions implemented. Environmental sampling of the NICU and Labour ward was performed. We employed WGS to differentiate phenotypically identical multidrug-resistant A.baumannii (MDRAB) strains from simultaneous intrahospital outbreaks in the adult Intensive Care Unit and NICU.
\end{abstract}

Results: Between 1/9/2012 and 31/12/2017 the surveillance included 2157 neonates. A total of 17 neonates had $A$. baumannii BSI, with the highest rate of 30.0 cases per 1000 bed-days in November 2012. Rectal screening samples were positive for A.baumannii-complex in 182 neonates reaching 119.6 per 1000 bed-days in July 2015. All 298 environmental cultures were negative. Two phenotypically identical MDRAB isolates from the simultaneous intrahospital outbreaks were differentiated using WGS, ruling out an inter-ward transmission. Adherence to stringent infection control measures decreased BSI cases but colonization remained persistent. With several relapses, the outbreak was ongoing for four years. No new A.baumannii BSI cases were registered after total environmental decontamination in the NICU in July 2015. Colonization reappeared and persisted until in November 2016 when the ward was temporarily closed, relocated and renovated. No A.baumannii cases were registered after the renovation.

Conclusion: The HAl surveillance system successfully detected and facilitated the control of the A.baumannii outbreak. Whole-genome sequencing was found to be a useful method for differentiation of phenotypically identical $A$. baumannii strains from the intrahospital outbreak. Only multi-modal infection control program, including closure, temporary relocation, and renovation of the ward, restricted the outbreak.

Keywords: Acinetobacter, Baumannii, Outbreak, Neonatal intensive care

\footnotetext{
* Correspondence: uga.dumpis@stradini.lv

'Pauls Stradins Clinical University Hospital, Riga, Latvia

University of Latvia, Riga, Latvia

Full list of author information is available at the end of the article
}

(c) The Author(s). 2019 Open Access This article is distributed under the terms of the Creative Commons Attribution 4.0 International License (http://creativecommons.org/licenses/by/4.0/), which permits unrestricted use, distribution, and reproduction in any medium, provided you give appropriate credit to the original author(s) and the source, provide a link to the Creative Commons license, and indicate if changes were made. The Creative Commons Public Domain Dedication waiver (http://creativecommons.org/publicdomain/zero/1.0/) applies to the data made available in this article, unless otherwise stated. 


\section{Introduction}

Acinetobacter baumannii has emerged worldwide as an important hospital-acquired infection (HAI) causing pathogen. [1,2] The bacteria can survive in the environment for months, thus making transmission difficult to prevent and control. [3] A.baumannii can colonize the human skin and gastrointestinal tract and thereby can cause HAIs. [4-6] Neonates admitted to Neonatal Intensive Care Unit (NICU) are at increased risk of contracting HAIs due to their immature immune system and frequent invasive manipulations. [7-9] Bloodstream infections (BSIs) caused by A.baumannii occur primarily in premature low-birth-weight infants. [8] In 4-year surveillance of device-associated HAIs in a NICU in Turkey A.baumannii was described as the main cause of device-associated HAIs. [10] HAIs outbreaks are associated with higher mortality, morbidity and increased hospital costs. [11-13] Surveillance of HAIs is essential for detection of the outbreaks and their containment. [11, 14-20] Standard typing methods during the surveillance and investigation of the outbreak were found to be useful in revealing relationships between isolates but could not to resolve differences between closely related strains. Whole-genome sequencing has shown to be more sensitive in discriminating between closely related strains, including from intra-hospital outbreaks. [21-24]

In this report, we describe how an implementation of the HAIs surveillance system for the first time in a PSCUH NICU in Latvia led to the rapid detection of an outbreak of A.baumannii. The objective of this report is to summarize the investigation and restriction of a continuous outbreak using whole-genome sequencing and multi-modal infection control interventions.

\section{Methods}

\section{Clinical setting}

Pauls Stradins Clinical University Hospital (PSCUH) is an 860-bed teaching hospital providing primary and tertiary-care to adults and neonatal patients with approximately 47,700 admissions accounting for 255,000 patient-days per year. The Neonatal Intensive Care Unit (NICU) with seven intensive care beds is providing care to approximately 250 critically ill neonates yearly, all transferred from PSCUH Labour ward. The NICU is open-plan divided into two sections - three and four beds in each. Until September 2012 PSCUH NICU did not have a HAIs surveillance system. In September 2012 a prospective neonatal HAI surveillance system was adapted and introduced [25] in the NICU including all patients admitted from the PSCUH Labour ward to the NICU.

\section{Epidemiological data}

Soon after the implementation of the HAIs surveillance system in September 2012, an A.baumannii outbreak was detected. A case definition of A.baumannii BSI was developed, using the definitions of the National Nosocomial Infections Surveillance Systems of the Centre for Disease Control and Prevention (CDC), adjusted for NICU patients. [25] Two months after the implementation of the surveillance system neonatal screening for A.baumannii colonization was initiated. Surveillance cultures of rectal swab specimens were obtained from all NICU patients at the admission and before the discharge. Colonization was defined as a rectal swab sample tested positive for A.baumannii-complex. Simultaneously samples from A.baumannii outbreak in the adult intensive care unit (ICU) with the phenotypically identical A.baumannii strain were collected. To resolve differences and exclude transmission between two wards situated in the same building, whole genome sequencing (WGS) was performed on matching A.baumannii samples isolated from adult ICU and NICU in the same outbreak period.

\section{Microbiology}

Blood cultures were processed using the BacT/ALERT automated system (bioMérieaux). When the system alerted a positive result, gram staining was performed and inoculated on blood, MacConkey, and egg-yolk salt agars. After $24 \mathrm{~h}$ all lactose negative colonies were inoculated on Kligler iron agar, sulfur indole motility medium, and Simmons citrate agar. Anal swab specimens were inoculated on Levine agar and MacConkey agar. Further inoculations of lactose negative gram-negative pathogens were performed as previously described. All non-fermenting pathogens were identified using either Vitek GN, Vitek2 automated system (bioMérieaux) or BBL Crystal enteric/non-fermenter ID kit (Becton Dickinson) and reported as A.baumannii-complex. Antimicrobial susceptibility testing was performed using disk-diffusion testing as recommended by The Clinical \& Laboratory Standards Institute (CLSI) for trimethoprim-sulfamethoxazole, amikacin, gentamicin, imipenem, ceftazidime, piperacillin-tazobactam, piperacillin, ampicillin-sulbactam.

\section{Whole-genome sequencing}

Sequencing was carried out at the Latvian Biomedical Research and Study Centre using Life Technologies Ion Torrent $^{\mathrm{TM}}$ PGM platform. [26] Invitrogen Qubit 2.0 Fluorometer (Invitrogen) was used to normalize genomic DNA concentration to $20 \mathrm{ng} / \mu \mathrm{l}$. Standard barcoded Fragment library preparation protocol with DNA input of $1 \mu \mathrm{g}$ and Ion Plus Fragment Library kit was used to prepare DNA libraries. Physical DNA fragmentation procedure was carried out using Covaris S220 focused-ultrasonicator (Covaris) with shearing parameters for $300 \mathrm{bp}$ long DNA fragments: peak incident power - 175; duty factor - 10\%; cycles per burst - 200; treatment time - 50s; temperature $7{ }^{\circ} \mathrm{C}$; water level S220-12; sample volume - $50 \mu \mathrm{l}$. Size 
selection was done using 1.5\% Dye Free Agarose gel cassettes on Blue Pippin (Labgene scientific) automated DNA size selection and collection device; targeted elution of 390 bp long fragments were chosen. Commercially available, platform-specific barcodes were used during library preparation to distinguish different samples (Ion Xpress ${ }^{\text {Tu }}$ Barcode Adapters). The final library quantification and the quality check were performed on Agilent 2100 Bioanalyzer using High Sensitivity DNA chips (Agilent Technologies). The libraries were diluted to a concentration of approximately 19 $\mathrm{pM}$ and each of the six libraries were pooled together. Ion OneTouch $^{\text {nv }} 200$ Template Kit v2 DL (Release: 12 September 2012, Publication No. MAN0006957) was used for template preparation and emulsion PCR. Ion $\mathrm{PGM}^{\mathrm{Tx}}$ Sequencing 300 Kit (Release: 6 September 2012, Publication No. MAN0007062) and standard sequencing protocol for Ion $318^{\text {tix }}$ chip type was used for the sequencing procedure. The predicted average coverage was $\sim 30 \mathrm{x}$.

\section{Whole-genome sequencing data analysis}

The reference genomes were searched in NCBI's GenBank using keywords '((Acinetobacter[Title]) AND "complete genome"[Title]) NOT plasmid[Title] NOT phage[Title] NOT bacteriophage[Title]'. As a result, 23 reference genomes were found from which 4 were excluded as duplicates. $200 \mathrm{bp}$ reads were generated from retained reference genomes with the depth of coverage 100. Sequenced samples and generated reads were mapped against reference genome TCDC-AB0715 (acc. no.: NC_017387.1) using tmap-3.4.1 mapping software. Mapping was performed using k-mer lookup algorithm (mapping procedure \#3 in tmap software). PCR duplicates were removed using Picard and local realignment around indels was performed using GATK-3.1-1. SNP calling was performed using GATK's UnifiedGenotyper SNP calling an algorithm. Further SNP filtering was applied with custom Python script retaining only SNPs with B-allele frequency at least 0.6 and depth of coverage at least 10 (statistics were calculated from VCF file using fields "AD" and "DP" respectively). Resulting SNPs were concatenated, creating genotypes for each sample. Phylogenetic analysis on resulting genotypes was performed using MEGA 5 software with the UPGMA algorithm and bootstrap on 500 replicates.

\section{Infection control interventions}

After identification of the A.baumannii outbreak in September 2012, an Infection Prevention and Control (IPC) team was formed in PSCUH from the local staff available (one IPC nurse, one Infectious Disease (ID) specialist, one junior doctor, one epidemiologist). The team visited NICU weekly reporting every new A.baumannii case back to the ward staff. Multi-modal infection control program was introduced: recommendations for patient and ward area improvement according to IPC principles were developed; hand hygiene procedures were reassessed and intensified; adherence to infection control measures was controlled by the IPC team; meetings with the hospital board were held; invasive manipulation indications and procedures were reviewed; patient screening for A.baumannii colonization at the admission and before the discharge was initiated; parenteral and enteral feeding guidelines and procedures were revised and re-implemented; environmental screening and decontamination was performed repeatedly. (Fig. 2).

\section{Environmental investigation}

Environmental cultures were collected from multiple high-touch surfaces in the NICU (e.g. computer keyboard, desks, patient monitors), medical devices (e.g. ultrasonography device, intubation equipment), feeding equipment (e.g. milk pumps, bottles, feeding mixtures), hands of the healthcare workers and environment in the ward (e.g. room air, tap aerators, water, clothing and sheets, disinfectant containers). The ventilation and air conditioning system was assessed, disassembled, revised and cultured. Extensive environmental sampling was performed in the Labour ward to exclude A.baumannii transmission considering that all neonates are admitted to the NICU from PSCUH Labour facilities.

\section{Results \\ Outbreak description}

In the surveillance, we included 2157 neonates admitted to PSCUH NICU from 1/9/2012 until 31/12/2017. In September 2012, because 13.3 A.baumannii BSI cases per 1000 patient days were detected, we initiated an outbreak investigation and implemented a multi-modal infection control program. From 1/9/2012 until 31/12/2017 applying case definitions we identified 17 A.baumannii BSIs, 70.6\% of infants had very low birth weight $(<1500 \mathrm{~g}$ according to the World Health Organization definition). In patients with BSIs mean birth weight was $1244.6 \mathrm{~g} \pm 732.2 \mathrm{~g}$, mean gestational age was $27.5 \pm 2.1$ weeks, $53 \%$ of the patients were male. Two patients with A.baumannii BSI died during the surveillance period. The highest incidence of A.baumannii BSIs was registered in November 2012 reaching 30.0 cases per 1000 patient days. From $1 / 11 / 2012$, when colonization screening was initiated, until 31/12/2017 we identified 182 A.baumannii colonization cases. The highest colonization rate was in July 2015 reaching 119.6 cases per 1000 patient days. An outbreak with several relapses was ongoing for four years. All patient rectal samples obtained before the admission in the NICU were negative for A.baumannii-complex. All A.baumannii isolates in the NICU had the same resistance pattern being resistant to trimethoprim/sulfamethoxazole, piperacillin, and gentamicin, except one A.baumannii isolate (Fig. 1 LAT_NICU_21.01.2014.) resistant to imipenem, piperacillin/tazobactam, ceftazidime, trimethoprim/sulfamethoxazole. This strain was phenotypically identical to the 


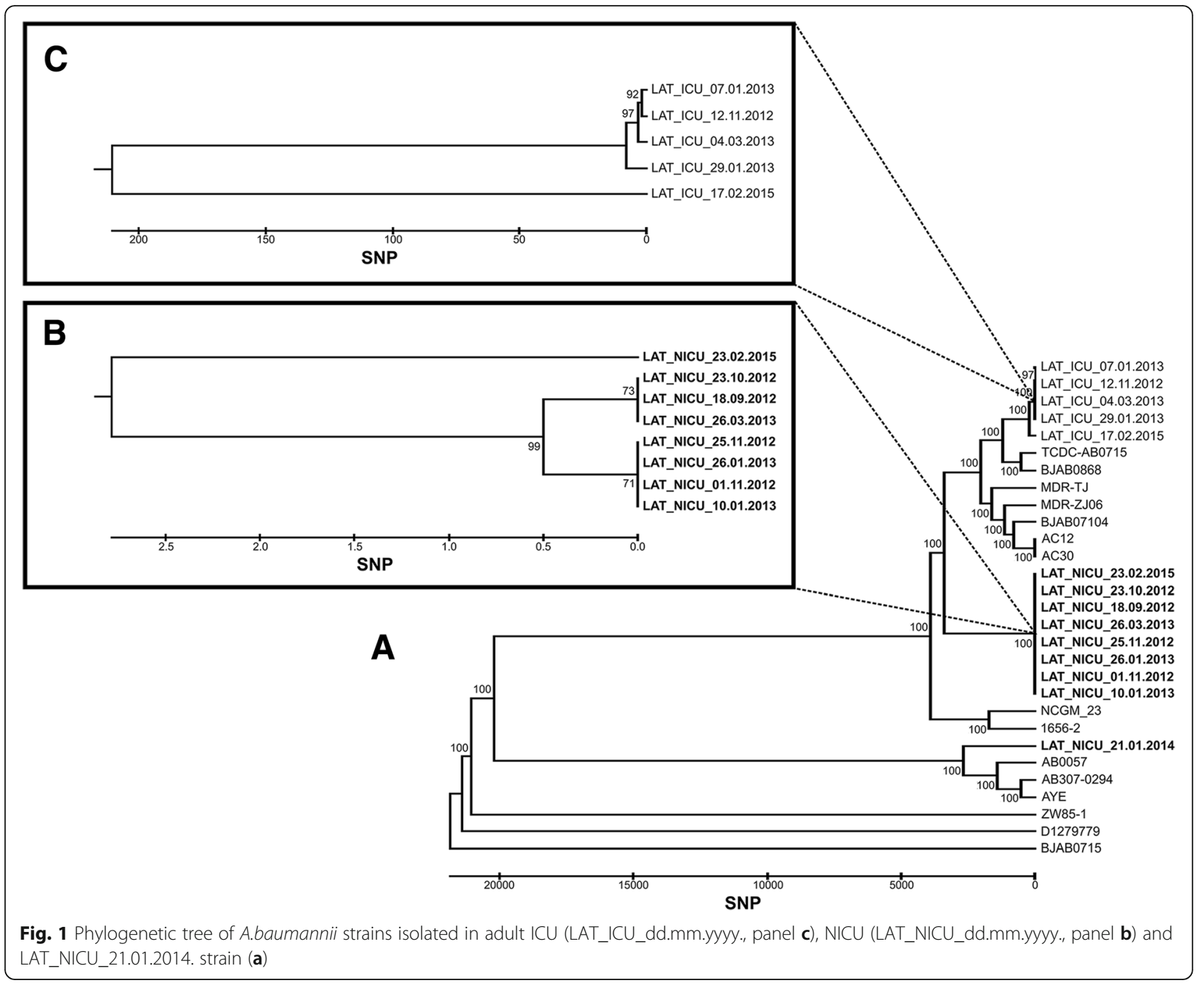

strains from the simultaneous multidrug-resistant A.baumannii (MDRAB) outbreak in the adult ICU located in the same hospital building.

\section{Infection control interventions}

In September 2012, after the detection of the outbreak, we did environmental assessment in the NICU and developed recommendations for patient and ward area improvement according to the IPC principles. (Fig. 2) The IPC team controlled adherence to the infection control measures during regular meetings with the NICU staff.

In October 2012 hand hygiene was intensified and educational workshops held for doctors and nurses; invasive procedures (intubation, central vascular catheterization (CVC), feeding with a nasogastric tube, parenteral feeding) were reviewed.

In November 2012 A.baumannii BSIs incidence was registered to reach 30.0 cases per 1000 bed-days. Rectal swab screenings for A.baumannii colonization at the admission and before the discharge were initiated; desks and medical documentation were relocated from the patient area; each patient's zone was equipped with individual equipment; room for feeding mixture and milk preparation with clean and potentially contaminated areas was allocated; tap aerators were removed and cultured. The infection control nurse participated in invasive manipulations and educated NICU staff and patients' family about the IPC procedures.

During December 2012 numerous workshops about invasive manipulations were held; central venous catheterization and umbilical catheterization indications were revised; the use of $\mathrm{CVC}$ was decreased; incubator humidifiers were not filled with water before the arrival of the patient anymore. The parenteral feeding mixture preparation guidelines were revised; the enteral feeding procedure was changed - sterile syringe was used before every new feeding episode.

In January 2013 parenteral feeding filters were introduced in the ward; weekly environmental disinfection in the NICU was initiated; the process of sterilization of 


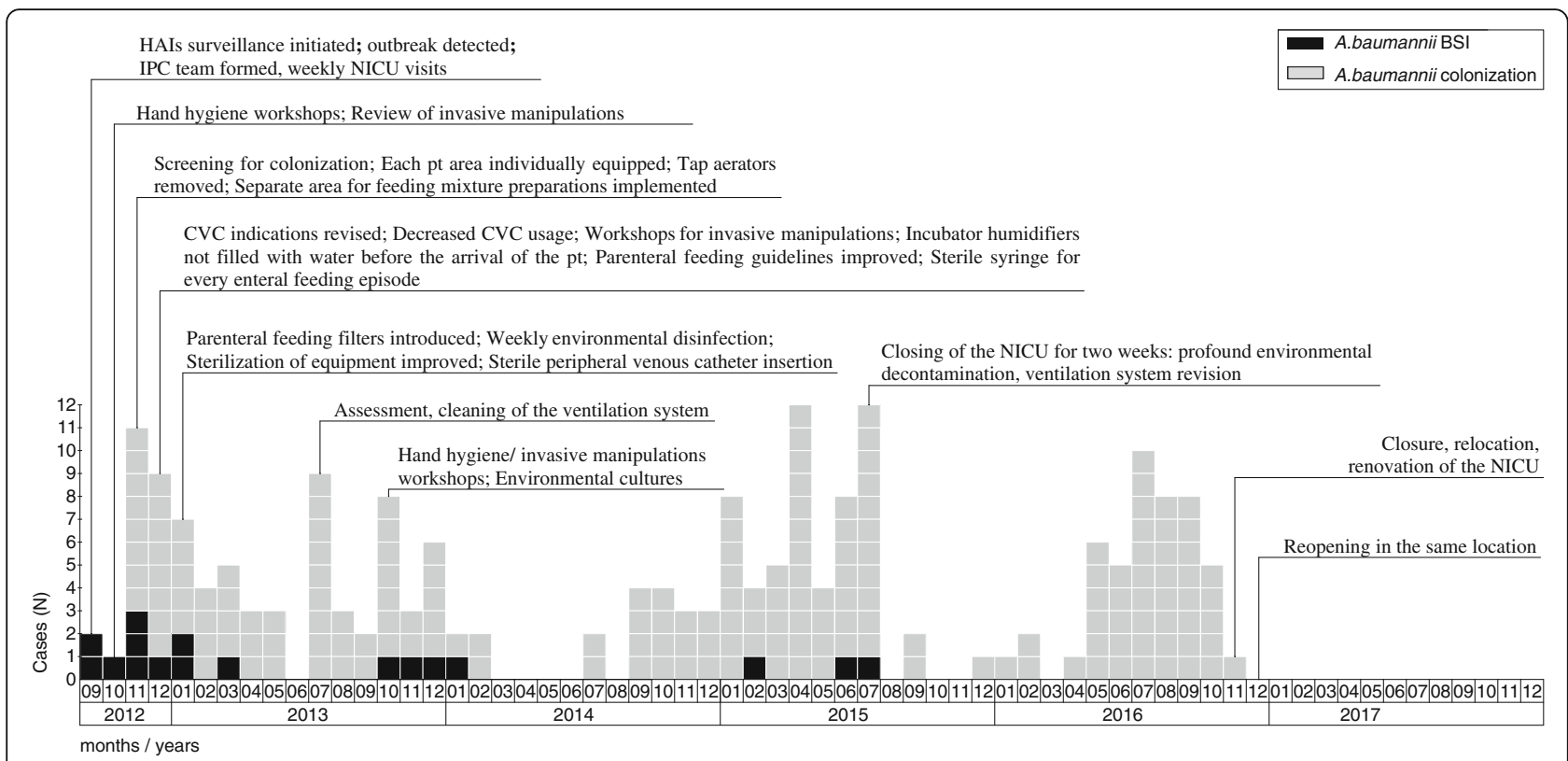

Fig. 2 A.baumannii BSI and colonization cases in relation to infection control interventions. BSI bloodstream infection, CVC central vascular catheter, N number, NICU Neonatal Intensive Care Unit, HAl hospital-acquired infection, IPC infection prevention and control, pt. patient

reusable equipment was re-evaluated and improved; sterile peripheral venous catheter insertion was implemented. As infection control interventions were intensified A.baumannii rates decreased. From April 2013 until October 2013 no new A.baumannii BSIs were registered. In addition, A.baumannii colonization cases decreased until no cases were registered in June 2013.

In July 2013 during the summer heat, A.baumannii colonization peaked again reaching 52.0 cases per 1000 bed-days. After the assessment of the risk factors, we identified that the increase of colonization cases was after the air conditioning system was turned on. Together with the Technical Department the ventilation system was assessed and cultured. All cultures came back negative for A.baumannii.

In October 2013 another case of A.baumannii BSI was detected after a six-month break. Re-assessment of the invasive manipulations and workshops of hand hygiene were re-implemented, the environment was recultured and meetings with the Hospital Board were held. Registered cases decreased due to intensified infection control measures. No new A.baumannii BSI cases were registered for more than a year from February 2014 until January 2015. But after the peak of colonization in January 2015 we detected a BSI in February 2015. Two more BSI cases were identified in June and July 2015. Nevertheless, the highest rate of A.baumannii colonization was detected in July 2015 reaching 119.6 cases per 1000 patient-days. The decision to close the NICU for two weeks for profound environmental decontamination and ventilation system revision was made. Complete closure with the renovation was not possible that time due to financial constraints. Since reopening and stricter infection control measures in the NICU, no new A.baumannii BSI cases were registered. Strengthened infection control interventions restricted A.baumannii BSI cases, but colonization re-emerged indicating that environmental source remained undetected.

In May 2016 colonization rates increased repeatedly reaching 89.0 cases per 1000 bed-days in July 2016 when air condition was used. The infection control activities were intensified again. Repeated meetings with the Hospital Board were held insisting on the closure of the NICU for ventilation system repair and renovation. In November 2016, the decision to close the ward was made. NICU was temporarily relocated, the ventilation system was disassembled, re-cultured and repaired. The renovation of the ward was ongoing for three weeks. During the relocation and after reopening of the ward in the same location no new A.baumannii cases were registered.

\section{Environmental sampling}

In total 298 environmental cultures were collected with extensive sampling on high-touch surfaces in NICU and Labour ward and were all negative for A.baumannii.

After the assessment of the risk factors, the ventilation system seemed to be the possible source of the outbreak. Thus together with the Technical Department, we performed a profound examination and complete disassembly of it. We identified that the ventilation system had numerous construction failures and it had not been cleaned nor examined. Also, periodically there were inappropriate room temperatures due to a malfunction of the air conditioner. 
The ventilation system was cleaned, disinfected, repaired and cultured repeatedly for A.baumannii- all cultures were negative.

\section{Whole-genome sequencing}

All A. baumannii isolates from the NICU had the same antimicrobial resistance pattern and were phenotypically identical, except one MDRAB causing BSI (Fig. 1 LAT_NICU_21.01.2014.) resistant to imipenem, piperacillin/ tazobactam, ceftazidime, trimethoprim/sulfamethoxazole. This strain was phenotypically identical to the strains isolated from simultaneous MDRAB outbreak in PSCUH adult ICU. Both wards are located in the same building so we suspected inter-ward transmission of the bacteria. To resolve the differences between these closely related strains, we performed WGS. It was carried out on five samples of MDRAB from the adult ICU matching retrospectively nine samples from the NICU (LAT_ICU_dd.mm.yyyy.) outbreak - two samples from each year $(2012,2013,2015)$ and a sample from 21/01/2014, identical to the strain from adult ICU. As the phenotypical pattern of the selected samples from the outbreak period suggested a single strain outbreak, except isolate LAT_NICU_21.01.2014, WGS was not performed to all A.baumannii isolates from NICU. The phylogenetic analysis of WGS identified three distinct clones. NICU isolates clustered together, while LAT_NICU_21.01.2014. phylogenetically was distinct from all other strains isolated from both - NICU and adult ICU - during the same period. Thus WGS excluded inter-ward transmission (Fig. 1). The MDRAB strain from NICU did not spread in the ward and was apparently contained by the intensified infection control measures.

\section{Discussion}

Here we report a rapid identification of an outbreak of $A$. baumannii resulting from an HAI surveillance system implementation for the first time in PSCUH NICU. We successfully used WGS to characterize an epidemiological pattern of the intrahospital outbreak of two phenotypically identical A.baumannii strains. The formation of the IPC team and implementation of the multi-modal infection control program, including renovation of the ward, were the key elements to contain the outbreak.

Previously published studies have shown that infection control measures are instrumental in the containment of the HAIs outbreaks. $[27,28]$ As soon as our outbreak was detected, the IPC team from the staff available at our hospital was formed and respective infection control interventions were intensified. We employed various infection control activities such as hand hygiene workshops, IPC education for the staff and patients families, environmental cleaning, etc. These interventions seemed to be effective only in reducing A.baumannii BSI cases.
Despite all the effort colonization cases re-emerged suggesting an environmental source. (Fig. 2).

Restriction of the spread of HAIs in NICU is challenging, especially if the outbreak is caused by A.baumannii because the bacteria can survive in the environment for long periods of time. [3] In some studies, investigators have managed to isolate the outbreak-causing pathogen from hygroscopic bandages [11], ventilator surfaces, bedside curtains, and a bed rail [29], but most of the studies have failed to identify the source. $[28,30]$ Even in the large scale Serratia marcescens outbreak in the NICU in Germany more than 600 environmental samples were taken and all were found to be negative. [31] We failed to identify the source after taking almost 300 environmental samples, though the pattern of the epidemic curve (Fig. 2) strongly indicated possible environmental contamination followed by further spread through contact transmission. Culture-based methods might not be sensitive enough to identify low-level environmental contamination. Real-time polymerase chain reaction (R-T PCR) assay has demonstrated better sensitivity. [32] Though the presence of the genomic DNA of A.baumannii could be associated with the identification of nonviable remains of the bacteria being present after decontamination, it could be helpful to identify the source. At that time R-T PCR assay was not available in our setting. An alternative explanation of the negative cultures in the NICU could be the reintroduction of bacteria from an outside source but no evidence to support this was found. All environmental samples from the Labour ward and rectal samples from patients before the admission were also negative. Thus, we were not able to confirm environmental contamination with positive cultures, even when the epidemiological curve (Fig. 2) suggested it.

After the assessment of the risk factors, we noticed that the colonization rate increased in the summer heat when the air conditioner was turned on. Significant shortcomings of the ventilation system were identified after assessment of the ward together with the Technical Department. The ventilation system was cleaned and cultured repeatedly. All cultures from the room air and ventilation were negative. Lack of finances led to delays in the renovation of the ward including complete disassembly and repair of the ventilation system. The decision on closure of the ward for two weeks with profound cleaning and culturing of the ventilation system was made. This strategy seemed to contain the A.baumannii BSIs and no new cases were detected since August 2015. Profound cleaning of the ward was described as a strategy in the control of A.baumannii in the previous study by Munier et al. [33] However, further colonization cases were identified even after this intervention. (Fig. 2). 
Colonization persisted and we still could not identify the point source. Since the general characteristics of the patients, including birth weight and gestational age, were similar over the surveillance period, we assume that those did not significantly affect the BSI and colonization rates. The source seemed to be the ventilation system despite the negative culturing results. Finally, the decision to close the ward for the renovation including repair of the ventilation system was made. Temporary closure, relocation, and renovation of the ward managed to eliminated cases of colonization. We believe that the unidentified environmental source was eradicated.

During an outbreak, it was important to characterize the genetic relationship between isolates. Phylogenetic relationship of the strains could be critical in guiding the infection control measures and restricting the outbreak. Whole-genome sequencing technology has been recognized to be more effective than the traditional methods for characterization of the outbreaks caused by Gram-negative bacteria. [34, 35] This was the first time WGS powered investigation was used in Latvia to resolve the intrahospital outbreak of A.baumannii. The phylogenetic analysis excluded inter-ward transmission between the adult ICU and NICU. A phenotypically identical but phylogenetically different MDRAB strain (LAT-NICU-21.01.2014 in Fig. 1) was identified in the NICU and was apparently contained by the infection control measures. This could also emphasize environmental contamination by the predominant strain.

\section{Conclusions}

We have described an outbreak in the NICU caused by $A$. baumannii contained with a multi-modal infection control program, including closure, temporary relocation, and renovation of the ward. Introduction of the HAI surveillance system was a crucial step towards timely identification and control of the outbreak. Whole-genome sequencing was found to be a useful method for tracking A.baumannii and differentiated phenotypically identical strains. We conclude that only multi-modal infection control interventions contained the outbreak and could be recommended in similar occasions.

\footnotetext{
Abbreviations

BSI: Bloodstream infection; CDC: Center for Disease Prevention and Control ; CLSI: Clinical \& Laboratory Standards Institute; CPAP: Continuous positive airway pressure; CVC: Central vascular catheters; EUCAST: The European Committee on Antimicrobial Susceptibility Testing; g: grams; HAl: Hospital-acquired infection; ICU: Intensive care unit; ID: Infectious disease; IPC: Infection prevention and control; MDRAB: Multidrug-resistant A.baumannii; N: Number; NICU: Neonatal intensive care unit; PSCUH: Pauls Stradins Clinical University Hospital; pt.: Patient; WGS: Whole-genome sequencing
}

\section{Funding}

Funded by the Latvian National Research Program Biomedicine for Public Health (BIOMEDICINE) 2014-2017.

\section{Availability of data and materials \\ The datasets used and/or analyzed during the current study are available from the corresponding author on reasonable request.}

\section{Authors' contributions}

Conception and design of study: DU, GA, DE; Acquisition of data: UV, ZIr, Zle, GA, SI, SM, BA, R-SI; Analysis and/or interpretation of data: DU, GA, DE, SM, SI, Zle, KJ, FD; Drafting the manuscript: DU, GA, FD, SI; Revising the manuscript critically for important intellectual content: DU, FD, DE. All authors read and approved the final manuscript.

Ethics approval and consent to participate

Since no patient personal information was collected and the surveillance was part of the hospital infection control and quality improvement program, approval of ethics committees was not considered necessary.

\section{Consent for publication}

Not applicable.

\section{Competing interests}

The authors declare that they have no competing interests.

\section{Publisher's Note}

Springer Nature remains neutral with regard to jurisdictional claims in published maps and institutional affiliations.

\section{Author details}

${ }^{1}$ Pauls Stradins Clinical University Hospital, Riga, Latvia. ${ }^{2}$ University of Latvia, Riga, Latvia. ${ }^{3}$ Latvian Biomedical Research and Study Center, Riga, Latvia.

${ }^{4}$ Center for Disease Prevention and Control, Riga, Latvia.

Received: 15 March 2019 Accepted: 13 May 2019

Published online: 22 May 2019

\section{References}

1. Corbella X, Montero A, Pujol M, Dominguez MA, Ayats J, Argerich MJ, et al. Emergence and rapid spread of carbapenem resistance during a large and sustained hospital outbreak of multiresistant Acinetobacter baumannii. J Clin Microbiol. 2000;38(11):4086-95.

2. Gales AC, Jones RN, Forward KR, Linares J, Sader HS, Verhoef J. Emerging importance of multidrug-resistant Acinetobacter species and Stenotrophomonas maltophilia as pathogens in seriously ill patients: geographic patterns, epidemiological features, and trends in the SENTRY antimicrobial surveillance program (1997-1999). Clinical infectious diseases : an official publication of the Infectious Diseases Society of America. 2001; 32(Suppl 2):S104-13.

3. Jawad A, Seifert H, Snelling AM, Heritage J, Hawkey PM. Survival of Acinetobacter baumannii on dry surfaces: comparison of outbreak and sporadic isolates. J Clin Microbiol. 1998;36(7):1938-41.

4. Struelens MJ, Carlier E, Maes N, Serruys E, Quint WG, van Belkum A. Nosocomial colonization and infection with multiresistant Acinetobacter baumannii: outbreak delineation using DNA macrorestriction analysis and PCR-fingerprinting. The Journal of hospital infection. 1993;25(1):15-32.

5. Smith A, Saiman L, Zhou J, Della-Latta P, Jia H, Graham PL 3rd. Concordance of gastrointestinal tract colonization and subsequent bloodstream infections with gram-negative bacilli in very low birth weight infants in the neonatal intensive care unit. Pediatr Infect Dis J. 2010;29(9):831-5.

6. Nagels B, Ritter E, Thomas P, Schulte-Wissermann H, Wirsing von Konig CH. Acinetobacter baumannii colonization in ventilated preterm infants. European journal of clinical microbiology \& infectious diseases : official publication of the European Society of Clinical Microbiology. 1998;17(1):37-40.

7. Goldmann DA, Freeman J, Durbin WA Jr. Nosocomial infection and death in a neonatal intensive care unit. J Infect Dis. 1983;147(4):635-41.

8. Huang YC, Su LH, Wu TL, Leu HS, Hsieh WS, Chang TM, et al. Outbreak of Acinetobacter baumannii bacteremia in a neonatal intensive care unit: clinical implications and genotyping analysis. Pediatr Infect Dis J. 2002; 21(12):1105-9. 
9. Gaynes RP, Martone WJ, Culver DH, Emori TG, Horan TC, Banerjee SN, et al. Comparison of rates of nosocomial infections in neonatal intensive care units in the United States. National Nosocomial Infections Surveillance System. Am J Med. 1991;91(3B):192S-6S.

10. Tekin R, Dal T, Pirinccioglu H, Oygucu SE. A 4-year surveillance of deviceassociated nosocomial infections in a neonatal intensive care unit. Pediatrics and neonatology. 2013;54(5):303-8.

11. Melamed R, Greenberg D, Porat N, Karplus M, Zmora E, Golan A, et al. Successful control of an Acinetobacter baumannii outbreak in a neonatal intensive care unit. The Journal of hospital infection. 2003;53(1):31-8.

12. Al Jarousha AM, El Jadba AH, Al Afifi AS, El Qouqa IA. Nosocomial multidrugresistant Acinetobacter baumannii in the neonatal intensive care unit in Gaza City. Palestine International journal of infectious diseases : IJID : official publication of the International Society for Infectious Diseases. 2009;13(5):623-8.

13. Thatrimontrichai A, Apisarnthanarak A, Chanvitan P, Janjindamai W, Dissaneevate S, Maneenil G. Risk factors and outcomes of carbapenemresistant Acinetobacter baumannii bacteremia in neonatal intensive care unit: a case-case-control study. Pediatr Infect Dis J. 2013;32(2):140-5.

14. Okpara AU, Maswoswe JJ. Emergence of multidrug-resistant isolates of Acinetobacter baumannii. Am J Hosp Pharm. 1994;51(21):2671-5.

15. Fournier $P E$, Richet $H$, Weinstein RA. The epidemiology and control of Acinetobacter baumannii in health care facilities. Clin Infect Dis. 2006;42(5):692-9.

16. Marques MB, Waites KB, Mangino JE, Hines BB, Moser SA. Genotypic investigation of multidrug-resistant Acinetobacter baumannii infections in a medical intensive care unit. J hospital infection. 1997;37(2):125-35.

17. Cordero L, Ayers LW, Davis K. Neonatal airway colonization with gramnegative bacilli: association with severity of bronchopulmonary dysplasia. Pediatr Infect Dis J. 1997;16(1):18-23.

18. Leistner R, Piening B, Gastmeier P, Geffers C, Schwab F. Nosocomial infections in very low birthweight infants in Germany: current data from the National Surveillance System NEO-KISS. Klinische Padiatrie. 2013;225(2):75-80.

19. Chan PC, Huang LM, Lin HC, Chang LY, Chen ML, Lu CY, et al. Control of an outbreak of pandrug-resistant Acinetobacter baumannii colonization and infection in a neonatal intensive care unit. Infection control and hospital epidemiology : the official journal of the Society of Hospital Epidemiologists of America. 2007;28(4):423-9.

20. Tsiatsiou O, losifidis E, Katragkou A, Dimou V, Sarafidis K, Karampatakis T, et al. Successful management of an outbreak due to carbapenem-resistant Acinetobacter baumannii in a neonatal intensive care unit. Eur J Pediatr. 2015;174(1):65-74.

21. Long SW, Beres SB, Olsen RJ, Musser JM. Absence of patient-to-patient Intrahospital transmission of Staphylococcus aureus as determined by whole-genome sequencing. mBio. 2014;5(5).

22. Reuter S, Ellington MJ, Cartwright EJ, Koser CU, Torok ME, Gouliouris T, et al. Rapid bacterial whole-genome sequencing to enhance diagnostic and public health microbiology. JAMA Intern Med. 2013;173(15):1397-404.

23. Koser CU, Holden MT, Ellington MJ, Cartwright EJ, Brown NM, Ogilvy-Stuart $\mathrm{AL}$, et al. Rapid whole-genome sequencing for investigation of a neonatal MRSA outbreak. N Engl J Med. 2012;366(24):2267-75.

24. Willems S, Kampmeier S, Bletz S, Kossow A, Kock R, Kipp F, et al. Wholegenome sequencing elucidates epidemiology of nosocomial clusters of Acinetobacter baumannii. J Clin Microbiol. 2016;54(9):2391-4.

25. Gastmeier P, Geffers C, Schwab F, Fitzner J, Obladen M, Ruden H. Development of a surveillance system for nosocomial infections: the component for neonatal intensive care units in Germany. J hospital infection. 2004;57(2):126-31.

26. Rothberg JM, Hinz W, Rearick TM, Schultz J, Mileski W, Davey M, et al. An integrated semiconductor device enabling non-optical genome sequencing. Nature. 2011:475(7356):348-52.

27. Liu WL, Liang HW, Lee MF, Lin HL, Lin YH, Chen CC, et al. The impact of inadequate terminal disinfection on an outbreak of imipenem-resistant Acinetobacter baumannii in an intensive care unit. PLoS One. 2014;9(9):e107975.

28. Ray A, Perez F, Beltramini AM, Jakubowycz M, Dimick P, Jacobs MR, et al. Use of vaporized hydrogen peroxide decontamination during an outbreak of multidrug-resistant Acinetobacter baumannii infection at a long-term acute care hospital. Infection control and hospital epidemiology : the official journal of the Society of Hospital Epidemiologists of America. 2010;31(12):1236-41.

29. Chang HL, Tang CH, Hsu YM, Wan L, Chang YF, Lin CT, et al. Nosocomial outbreak of infection with multidrug-resistant Acinetobacter baumannii in a medical center in Taiwan. Infection control and hospital epidemiology : the official journal of the Society of Hospital Epidemiologists of America. 2009;30(1):34-8.
30. Cristina ML, Spagnolo AM, Ottria G, Sartini M, Orlando P, Perdelli F, et al. Spread of multidrug carbapenem-resistant Acinetobacter baumannii in different wards of an Italian hospital. Am J Infect Control. 2011;39(9):790-4.

31. Gastmeier P. Serratia marcescens: an outbreak experience. Front Microbiol. 2014;5:81.

32. McConnell MJ, Perez-Ordonez A, Perez-Romero P, Valencia R, Lepe JA, Vazquez-Barba I, et al. Quantitative real-time PCR for detection of Acinetobacter baumannii colonization in the hospital environment. J Clin Microbiol. 2012;50(4):1412-4.

33. Munier AL, Biard L, Legrand M, Rousseau C, Lafaurie M, Donay JL, et al. Incidence, risk factors and outcome of multi-drug resistant Acinetobacter baumannii nosocomial infections during an outbreak in a burn unit. International journal of infectious diseases : IJID : official publication of the International Society for Infectious Diseases. 2018;79:179-84.

34. Didelot X, Bowden R, Wilson DJ, Peto TE, Crook DW. Transforming clinical microbiology with bacterial genome sequencing. Nat Rev Genet. 2012;13(9): 601-12.

35. Punina NV, Makridakis NM, Remnev MA, Topunov AF. Whole-genome sequencing targets drug-resistant bacterial infections. Human genomics. 2015;9:19.

\section{Ready to submit your research? Choose BMC and benefit from:}

- fast, convenient online submission

- thorough peer review by experienced researchers in your field

- rapid publication on acceptance

- support for research data, including large and complex data types

- gold Open Access which fosters wider collaboration and increased citations

- maximum visibility for your research: over $100 \mathrm{M}$ website views per year

At BMC, research is always in progress.

Learn more biomedcentral.com/submissions 